\title{
Dasatinib: the emerging evidence of its potential in the treatment of chronic myeloid leukemia
}

\author{
Sonya Haslam
}

Core Medical Publishing, Knutsford, UK

\begin{abstract}
Introduction: Current therapy options for chronic myeloid leukemia (CML) include conventional chemotherapy, allogeneic stem cell transplant, interferon-alfa, and imatinib mesylate, which has recently achieved gold standard status. Although the majority of patients initially respond well to treatment with imatinib, wider clinical experience with this drug has resulted in the development of imatinib resistance being increasingly documented. There is therefore an unmet medical need for novel therapies to override imatinib resistance in CML.
\end{abstract}

Aims: This review summarizes the emerging evidence for the potential use of dasatinib in the treatment of imatinib-resistant CML.

Disease and treatment: Dasatinib is a novel small molecule that has shown potent antileukemic activity in imatinib-resistant cell lines, malignant marrow cells isolated from patients with imatinib-resistant CML, and in mouse xenograft models of imatinib-resistant CML. Preliminary data from an initial phase I dose escalation trial have been encouraging, indicating that dasatinib is generally well tolerated and produces hematologic and cytogenetic responses in patients with imatinib-resistant CML in all phases of the disease. The maximum tolerated dose (MTD) has not yet been reached, and dose escalation continues to determine the dose range that yields optimal results.

Profile: Although dasatinib is still in the early stages of development, the potential impact of this molecule on the treatment of CML could be revolutionary, not only providing a much needed treatment option for patients with imatinib-resistant CML, but also, combined with imatinib, could possibly prove useful in delaying the onset of resistance to treatment. Furthermore, combined with other agents active in $\mathrm{CML}$, dasatinib could have potential utility in purging residual leukemic cells in patients whose disease is controlled by imatinib.

Key words: dasatinib, BMS-354825, BCR-ABL, SRC-ABL kinase inhibitor, chronic myeloid leukemia (CML), imatinib resistance, treatment, evidence, outcomes

\section{Core emerging evidence summary for dasatinib in imatinib-resistant chronic myeloid leukemia}

\begin{tabular}{|ll|}
\hline Outcome measure & Emerging evidence \\
\hline Hematologic response & Achieved in the majority of patients, regardless of stage of disease \\
Cytogenic response & $\begin{array}{l}\text { Achieved in substantial numbers of patients, even though the optimal dose has not yet been } \\
\text { determined } \\
\text { Molecular response }\end{array}$ \\
& $\begin{array}{l}1-2 \text { log reduction in BCR-ABL transcripts associated with major cytogenetic responses in patients } \\
\text { with chronic phase CML }\end{array}$ \\
\hline
\end{tabular}




\section{Scope, aims, and objectives}

Dasatinib (BMS-354825; Bristol-Myers Squibb) is a small molecule investigational drug, which is currently in phase I development to override imatinib resistance in chronic myeloid leukemia (CML). This review summarizes the disease background, current therapy options, and unmet medical needs, and examines the emerging evidence for the potential use of dasatinib in the treatment of imatinib-resistant CML.

\section{Methods}

The English language medical literature was searched on February 8, 2005 in the following databases:

- PubMed, http://www.ncbi.nlm.nih.gov/entrez/query.fcgi

- EMBASE, http://www.datastarweb.com

- Database of Abstracts of Reviews of Effectiveness (DARE), NHS Economic Evaluations Database (NHSEED), Health Technology Assessment (HTA), http://www.york.ac.uk/inst/crd/darehp.htm (all fields searched in all three databases together)

- NHS HTA, http://www.ncchta.org

- National Guideline Clearing House, http://wwwguideline.gov

- Cochrane Database of Systematic Reviews (CDSR), http://cochrane.org/index0.htm

- Clinical Evidence (BMJ), http://www.clinicalevidence.com

- EBM reviews (ACP Journal Club), http://www.acpjc.org.

The search terms used were "BMS-354825" OR "BMS354825", and the cut-off dates were from the beginning of the database to the date of the search.

A total of six articles were identified: three preclinical articles, and three editorial/opinion articles. No relevant systematic reviews or treatment guidelines involving dasatinib were identified.

The proceedings of the following oncology society meetings were also searched for relevant abstracts, again using the search terms "BMS-354825" OR "BMS354825":

- American Society of Hematology (ASH) 2004

- American Society for Clinical Oncology (ASCO) 2004 and 2005

- American Association for Cancer Research (AACR) 2004

- European Society for Medical Oncology (ESMO) 2004

- European Hematology Association (EHA) 2004.

A total of 23 abstracts was identified, seven of which were disregarded on the basis of not concerning dasatinib $(n=1)$ or not being relevant to the treatment of $\mathrm{CML}(n=6)$. Among the relevant abstracts, nine reported on the outcomes of preclinical studies, and seven on preliminary clinical data (Table 1).

\begin{tabular}{|lcc|}
\begin{tabular}{l} 
Table 1 \\
\multirow{2}{*}{$\begin{array}{l}\text { phase I only) } \\
\text { phidence base included in the review (preclinical and }\end{array}$} \\
\cline { 2 - 3 } Category
\end{tabular} & \multicolumn{2}{c|}{ Number of records } \\
\hline Full papers & Abstracts \\
\hline Initial search & 6 & 23 \\
records excluded & 0 & 7 \\
records included & 6 & 16 \\
\hline
\end{tabular}

\section{Disease overview}

CML, also known as chronic myelogenous leukemia, is a clonal myeloproliferative disorder that originates from a single abnormal hematopoietic stem cell (Faderl et al. 1999; John et al. 2004; Stone 2004). Myeloid progenitors expand at various stages of maturation and are released prematurely from the bone marrow into the peripheral blood, subsequently infiltrating extramedullary sites, particularly the spleen. In the advanced stages of the disease (accelerated phase or blast crisis) the liver and lymph nodes may also become involved.

\section{Epidemiology}

CML accounts for around $15 \%$ of all adult leukemias and is slightly more common in males than females (ratio 1.3:1). The incidence of CML is estimated at 1-2 new cases per 100000 per annum (Maness \& McSweeney 2004), and increases with advancing age (Fig. 1). The median age at CML diagnosis is $45-55$ years, although $12-30 \%$ of patients present after the age of 60 years, and $10 \%$ present before their 20th birthday (Cortes et al. 1996, 1997; Faderl et al. 1999; Hill \& Meehan 1999; Jemal et al. 2002; Besa 2004).

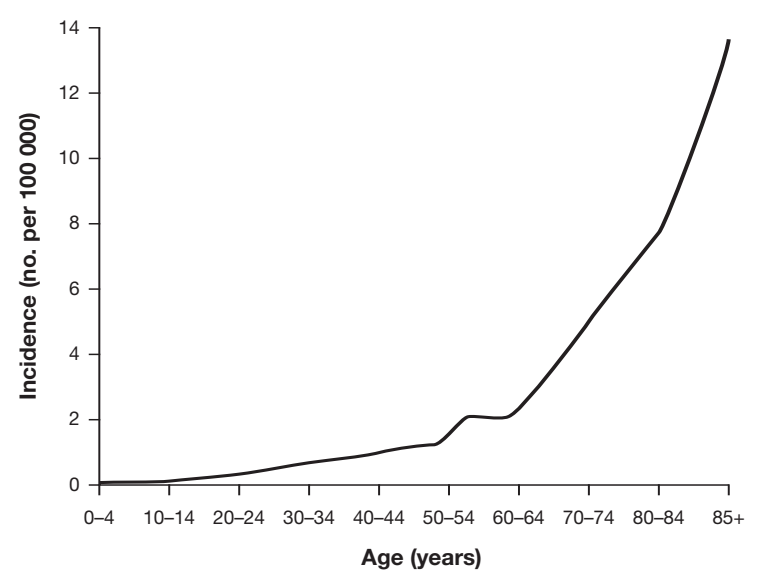

Fig. 1 | Age-specific incidence rate of chronic myeloid leukemia (CML) (1995-1998) increases with age. National Cancer Institute Surveillance Epidemiology and End Results (SEER) data (Table XIII-6, chronic myeloid leukemia), which is publicly available at http://seer.cancer.gov/csr/1973_1999/leukemia.pdf; graph adapted from the Leukemia and Lymphoma Society website: http://www.leukemia-lymphoma.org/all_mat_detail.adp?item_ $\mathrm{id}=2119$ \&sort_order $=48$ cat_id $=1209$ 


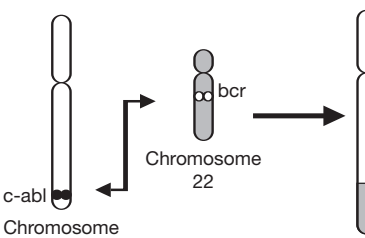

Fig. 2 | The Philadelphia translocation: a balanced reciprocal translocation between chromosomes $9 \mathrm{q}$ and $22 \mathrm{q}$, resulting in the creation of the bcr-abl fusion gene

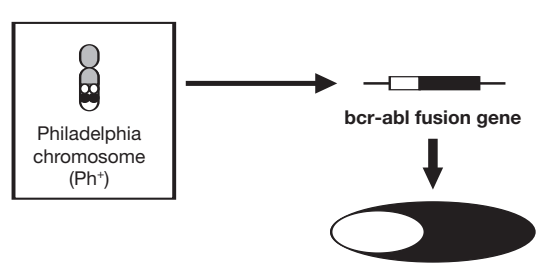

BCR-ABL oncoprotein with constitutive tyrosine kinase activity

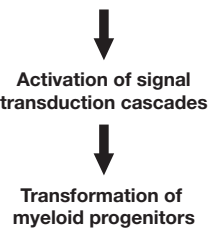

\section{Molecular basis of $C M L$}

At the molecular level CML is caused by the constitutive expression of the bcr-abl oncogene which is created by a balanced reciprocal translocation between chromosomes $9 q$ and 22q (Fig. 2) (Nowell \& Hungerford 1960; Rowley 1973; Faderl et al. 1999). This translocation, known as the Philadelphia translocation, results in the joining of the $3^{\prime}$ segment of the abl (Ableson leukemia virus) gene on $9 q 34$ and the $5^{\prime}$ segment of the bcr (breakpoint cluster region) gene on 22q11, to form the bcr-abl hybrid fusion gene carried on a shortened chromosome 22. This shortened chromosome 22, known as the Philadelphia chromosome $(\mathrm{Ph}+)$, is the hallmark of $\mathrm{CML}$.

The BCR-ABL oncoprotein is a deregulated nonreceptor tyrosine kinase with increased activity compared with the 'wild-type' $A B L$ kinase. Constitutive expression of BCR-ABL leads to malignant transformation by interfering with the signal transduction pathways involved in the control of apoptosis, cellular proliferation, and adherence of CML progenitors to bone marrow stromal elements (Goldman 1997; Faderl et al. 1999; John et al. 2004).

In $50-80 \%$ of $C M L$ cases, disease progression is associated with the acquisition of additional cytogenetic and molecular genetic changes, which often precede hematologic and clinical manifestations. Minor cytogenetic changes include monosomies of chromosomes 7 and 17 (one chromosome missing), trisomies of chromosomes 17 and 21 (an extra chromosome), and a translocation between chromosomes 3 and 12 [t(3;12)(q26,q22)] (Mitelman 1993). Major cytogenetic changes include trisomy 8 , isochromosome 17q, trisomy 19, and an additional Philadelphia chromosome (disomy $\mathrm{Ph}+$ ) (Kantarjian et al. 1987; Derderian et al. 1993; Mitelman 1993). The most frequent molecular abnormalities are alterations to the tumor suppressor, p53 (Ahuja et al. 1989).

\section{Signs and symptoms}

The onset of CML is associated with signs and symptoms that usually develop gradually. Most patients experience fatigue and reduced exercise tolerance due to anemia. Discomfort on the left side of the abdomen is also a frequent complaint as a result of splenomegaly. In addition, patients with CML often develop a hypermetabolic state, which is associated with fever, excessive sweating, and weight loss (Faderl et al. 1999; Hill \& Meehan 1999; Besa 2004).

\section{Disease staging}

CML follows a triphasic clinical course (Fig. 3). Initially there is the chronic phase, in which the disease is often indolent, followed by the accelerated phase, and subsequent progression to the terminal phase, known as blast crisis (Faderl et al. 1999; Hill \& Meehan 1999; Maness \& McSweeney 2004).

\section{Chronic phase}

The chronic phase is characterized by $<5 \%$ blasts (immature myeloid leukemic cells) in the blood and bone marrow; no malignant cells outside of the blood, bone marrow, or spleen; and absent/mild symptoms of CML. Most patients ( $80 \%)$ are in the chronic phase at diagnosis. The median duration of the chronic phase is $3-5$ years, before progression to the accelerated phase.

\section{Accelerated phase}

The accelerated phase is defined by the presence of one or more of the following: persistent presence of $10-30 \%$ blasts in the blood and bone marrow; white blood cell count $>50000 / \mu \mathrm{L}$ with an increase or decrease in platelets and low red blood cell count despite treatment; progressive splenomegaly; involvement of

\begin{tabular}{|c|c|c|}
\hline $\begin{array}{l}\text { Chronic } \\
\text { phase }\end{array}$ & $\begin{array}{l}\text { Accelerated } \\
\text { phase }\end{array}$ & $\begin{array}{l}\text { Blast } \\
\text { crisis }\end{array}$ \\
\hline \multirow[t]{2}{*}{$<5 \%$ blasts } & $10-30 \%$ blasts & \multirow[t]{2}{*}{$>30 \%$ blasts } \\
\hline & $\begin{array}{l}\text { Karyoty } \\
\text { evoluti }\end{array}$ & \\
\hline $\begin{array}{l}\text { - Often asymptomatic } \\
\text { - Fatigue } \\
\text { - Weight loss } \\
\text { - Abdominal pain } \\
\text { - Splenomegaly }\end{array}$ & $\begin{array}{l}\text { - Fever } \\
\text { - Fatigue } \\
\text { - Bone pain } \\
\text { - Progressive splenomegaly } \\
\text { - Chloroma }\end{array}$ & $\begin{array}{l}\text { - Fever } \\
\text { - Fatigue } \\
\text { - Bone pain } \\
\text { - Progressive splenomegaly } \\
\text { - Chloroma }\end{array}$ \\
\hline $\begin{array}{l}\text { Median duration } \\
3-5 \text { years }\end{array}$ & $\begin{array}{l}\text { Median duration } \\
6-9 \text { months }\end{array}$ & $\begin{array}{l}\text { Median survival } \\
\text { 3-6 months }\end{array}$ \\
\hline
\end{tabular}

Fig. 3 | Chronic myeloid leukemia: triphasic clinical course 
organs other than the bone marrow or spleen; acquired cytogenetic abnormalities additional to the Philadelphia chromosome; and persistent, unexplained fever or bone pain. The accelerated phase of $\mathrm{CML}$ is generally short-lived, with progression to blast crisis within a median of 6-9 months.

\section{Blast crisis}

The final stage of CML, blast crisis, is defined by the presence of $>30 \%$ blasts in the blood and bone marrow. These immature leukemic blasts may form tumor masses in the bone or lymph nodes. At this stage, the disease is considered to have progressed to an aggressive, acute form of leukemia that is rapidly fatal, with a median survival of 3-6 months. In one-third of cases, the blasts develop a lymphoid morphology and begin to express lymphoid markers, whereas in the remaining two-thirds the blasts have a phenotype similar to that of acute myeloblastic leukemia (Griffin et al. 1983). It is important to distinguish between these two groups of patients because those whose leukemia is in lymphoid blast crisis (Ph+ $\mathrm{ALL}$ ) can respond to treatment with regimens that are active against acute lymphoid leukemia (Derderian et al. 1993), whereas patients with myeloid morphology do not.

\section{Diagnosis}

Approximately $50 \%$ of patients with $\mathrm{CML}$ are asymptomatic at diagnosis; their disease being discovered after routine blood films show leukocytosis. Initial evaluations typically involve a physical examination and morphologic review and immunotyping of the blood, bone marrow, and, where appropriate, a lymph node or spleen biopsy. However, a confirmed diagnosis of CML depends on demonstrating the presence of the $\mathrm{Ph}+$ chromosome cytogenetically in the bone marrow (detected in 95\% of patients), or detecting the presence of the bcr-abl oncogene by polymerase chain reaction (PCR), fluorescent in situ hybridization (FISH), or Southern analysis; detecting the BCR-ABL transcript by RT-PCR or Northern analysis; or demonstrating the presence of the BCR-ABL oncoprotein by Western analysis (Faderl et al. 1999; Hill \& Meehan 1999; Vardiman et al. 2001).

\section{Evaluation of response}

Response to therapy for CML can be evaluated on three levels. In increasing order of remission depth these are hematologic responses, cytogenetic responses, and molecular responses.

A complete hematologic response refers to normalization of blood counts lasting for at least 4 weeks. However, patients who achieve complete hematologic responses may still have detectable levels of $\mathrm{Ph}+$ cells. A complete cytogenetic response refers to the absence of $\mathrm{Ph}+$ cells on cytogenetic analysis. For patients who achieve complete cytogenetic responses, a more sensitive measurement of residual disease may be conducted using BCR$A B L$ transcripts, detected by reverse transcriptase PCR (RT-PCR), as a marker for residual disease. Elimination of residual disease to levels below the limit of detection by RT-PCR is known as a complete molecular remission. Incomplete cytogenetic responses are referred to as major or minor responses according to the percentages of $\mathrm{Ph}+$ cells detected.

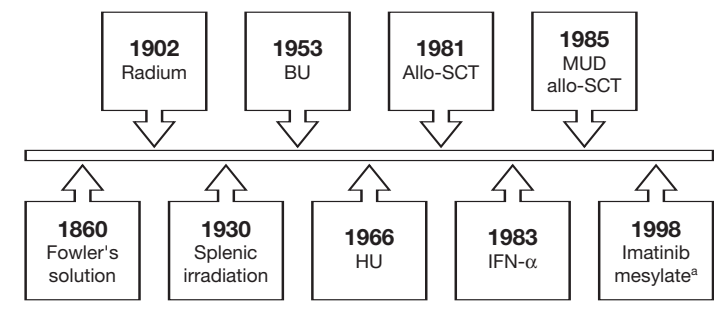

aPhase I trial initiated

Fig. 4 | Advances in the treatment of chronic myeloid leukemia: an historical perspective. allo-SCT, allogeneic stem cell transplant; $\mathrm{BU}$, busulfan; HU, hydroxyurea; IFN- $\alpha$, interferon-alfa; MUD, matched unrelated donor

\section{Goals of therapy}

The goal of therapy usually depends upon disease phase. For patients with chronic phase CML the principal goal of therapy has been to attain a complete cytogenetic response, as this has consistently been correlated with prolonged survival (Kantarjian et al. 1995, 2004; Clift \& Storb 1996; Guilhot et al. 1997; ICSGCML 1998; Clift et al. 1999; Stone 2004). However, molecular responses are likely to become the new goal of therapy for these patients, with the aim of further improving response duration and overall survival. Other important objectives of therapy for patients in the chronic phase include normalizing blood counts (complete hematologic responses), controlling the signs and symptoms of CML, and delaying disease progression to the accelerated phase or blast crisis.

In contrast, for patients in the accelerated phase or blast crisis, the primary goal of therapy is often to reestablish the chronic phase. Regardless of therapy the prognosis for patients with accelerated or blast phase CML is currently poor (Shah et al. 2004a; Travis 2004).

\section{Current therapy options}

Considerable advances have been made in the treatment of CML since the first patients were treated with Fowler's solution in the 1860s (Fig. 4).

\section{Chemotherapy}

Busulfan, an alkylating agent introduced in the 1950s, reduces white blood cell counts and disease-related signs and symptoms, but does not produce a complete cytogenetic response, or significantly delay disease progression. In addition, busulfan is poorly tolerated and is associated with serious adverse events, such as myelosuppression and pulmonary/hepatic/cardiac fibrosis (Hehlmann et al. 1993).

Treatment with hydroxyurea, a ribonucleotide reductase inhibitor, can achieve hematologic remissions and reduce splenomegaly, and offers a survival advantage over busulfan. Hydroxyurea is also better tolerated than busulfan, particularly with regard to bone marrow recovery. However, like busulfan, hydroxyurea produces neither a complete cytogenetic remission, nor a significant delay in disease progression to the accelerated phase or blast crisis (Hehlmann et al. 1993). 
The investigational drug, homoharringtonine, a plant alkaloid protein synthesis inhibitor, is also active in CML as monotherapy and in combination with cytarabine (Kantarjian et al. 2000, 2001). In patients with late chronic phase CML (diagnosed for $>12$ months) treatment with homoharringtonine produced complete hematologic and cytogenetic responses in 72 and 31\%, respectively (O'Brien et al. 1995). However, high doses and short infusion schedules of homoharringtonine are associated with serious cardiovascular complications, including hypotension and cardiac arrhythmias (Kantarjian et al. 2000, 2001). Consequently homoharringtonine has not received approval for the treatment of CML.

\section{Allogeneic stem cell transplantation}

Allogeneic hematopoietic stem cell transplantation (allo-SCT) is currently the only treatment with long-term data demonstrating curative potential in CML (Stone 2004). This is due to the graftversus-leukemia (GVL) effect, an immunotherapeutic phenomenon in which allogeneic $\mathrm{T}$ and NK cells recognize and destroy tumor cells (Bleakley \& Riddell 2004).

Up to $70 \%$ of patients with early chronic phase CML are cured following allo-SCT (Stone 2004). However, the probability of longterm survival is substantially reduced in patients who are transplanted in the accelerated or blast phases of the disease (Clift \& Storb 1996; Horowitz et al. 1996), and progressively worsens with advancing age (Clift \& Storb 1996; Gratwohl \& Hermans 1996; Gratwohl et al. 1998). Furthermore, allo-SCT procedures are associated with significant morbidity [graft-versus-host disease (GVHD) and infections], and high rates of treatment-related mortality (20-40\%) (Silver et al. 1999). An HLA-matched, related donor is optimal, but is available for $<30 \%$ of patients (Clift \& Storb 1996; Horowitz et al. 1996). In the absence of an HLA-matched, related donor, an HLA-matched, unrelated donor transplant may be considered, but is usually associated with increased morbidity (GVHD and infections, including cytomegalovirus reactivation) and an increased risk of treatment-related mortality, compared with an HLA-matched, related donor transplant (Hansen et al. 1998; Weisdorf et al. 2002).

Considering the current evidence, the best candidates for allo-SCT would therefore appear to be young patients with recently diagnosed, early-stage disease for whom matched, related donors are available. Consequently, $<40 \%$ of patients with $\mathrm{CML}$ are eligible for allo-SCT procedures, despite its curative potential. However, recently developed reduced-intensity conditioning regimens for
allo-SCT have been associated with reduced morbidity and mortality risks, and are likely to extend this potentially curative procedure to patients previously considered ineligible because of age and/or advanced disease (i.e. CML in the accelerated phase or blast crisis) (Or et al. 2003).

\section{Interferon}

The mechanism of action of interferon-alfa (IFN-alfa) is poorly understood. For patients with chronic phase CML, IFN-alfa produces complete cytogenetic responses in $8-38 \%$ of patients (ICSGCML 1998), and significantly prolongs survival compared with hydroxyurea or busulfan (Hehlmann et al. 1994; ICSGCML 1994). Furthermore, patients who achieve major cytogenetic responses following treatment with IFN-alfa survive longer, compared with those who fail to achieve a major cytogenetic response (Kantarjian et al. 1995). In common with other treatments for CML, IFN-alfa is most effective in the chronic phase of the disease (Kantarjian et al. 1998).

In a randomized study comparing the safety and efficacy of IFNalfa + cytarabine vs IFN-alfa monotherapy in patients with chronic phase CML, the addition of cytarabine was associated with significantly higher complete hematologic response rates (66 vs $55 \%)$, major cytogenetic response rates (41 vs $24 \%$ ), and significantly longer survival (3-year survival rates: 85.7 vs $79.1 \%$ ) (Guilhot et al. 1997).

In a long-term follow-up of 512 patients with CML treated with IFN-alfa + cytarabine between 1981 and 1995, 27\% achieved complete cytogenetic remissions, and among those patients achieving complete cytogenetic remissions, $78 \%$ achieved 10 -year survival. This confirms the importance of complete cytogenetic remission as a therapeutic goal in CML (Kantarjian et al. 1995).

The major drawback of IFN-based therapy is its poor tolerability profile: $10-25 \%$ of patients discontinue treatment due to flu-like syndrome, anorexia, and depression (ICSGCML 1994; Guilhot et al. 1997). However, despite its poor tolerability profile, IFN-alfa + cytarabine was the standard of care for many years before the introduction of imatinib mesylate, and is still used today.

\section{Imatinib mesylate}

The BCR-ABL oncoprotein is the most directly relevant therapeutic target in $\mathrm{CML}$. Imatinib mesylate, a small molecule developed specifically to inhibit BCR-ABL kinase activity, has revolutionized the treatment of $\mathrm{CML}$, and is currently the gold

Table 2 | Imatinib: phase II responses in treatment of chronic myeloid leukemia

\begin{tabular}{|c|c|c|c|c|c|}
\hline \multirow[b]{3}{*}{ Response } & \multicolumn{5}{|c|}{ Phase of disease } \\
\hline & \multirow{2}{*}{$\begin{array}{c}\text { Chronic } \\
\text { (Kantarjian et al. 2002) } \\
400 \mathrm{mg} / \text { day }\end{array}$} & \multicolumn{2}{|c|}{$\begin{array}{c}\text { Accelerated } \\
\text { (Talpaz et al. 2002) }\end{array}$} & \multicolumn{2}{|c|}{$\begin{array}{c}\text { Blast crisis } \\
\text { (Sawyers et al. 2002) }\end{array}$} \\
\hline & & $400 \mathrm{mg} /$ day & $600 \mathrm{mg} /$ day & $400 \mathrm{mg} /$ day & $600 \mathrm{mg} /$ day \\
\hline Complete hematologic & $95 \%$ & $27 \%$ & $37 \%$ & $0 \%$ & $9 \%$ \\
\hline Complete cytogenetic & $41 \%$ & $11 \%$ & $19 \%$ & $3 \%$ & $8 \%$ \\
\hline Major cytogenetic & $60 \%$ & $16 \%$ & $28 \%$ & $6 \%$ & $18 \%$ \\
\hline Minor cytogenetic & $5 \%$ & $8 \%$ & $6 \%$ & $3 \%$ & $2 \%$ \\
\hline
\end{tabular}


Table 3 | IRIS trial: phase III responses

\begin{tabular}{|lccc|}
\hline Category & Imatinib & $\begin{array}{c}\text { IFN-alfa/ } \\
\text { cytarabine }\end{array}$ & $P$ \\
\hline Complete hematologic response & $95.5 \%$ & $55.5 \%$ & $<0.001$ \\
Complete cytogenetic response & $73.8 \%$ & $8.5 \%$ & $<0.001$ \\
$\begin{array}{l}\text { Molecular responses (3-log } \\
\text { reduction in BCR-ABL } \\
\text { transcripts at 12 months) }\end{array}$ & $39 \%$ & $2 \%$ & $<0.001$ \\
Survival & & & \\
\hline IFN-alfa, interferon-alfa. & imatinib over IFN-alfa/cytarabine & \\
\hline
\end{tabular}

standard treatment for all phases of CML (Druker et al. 1996, 2001a,b; Peggs \& Mackinnon 2003). Phase II studies confirmed the ability of imatinib to produce both complete hematologic and cytogenetic remissions in patients in all phases of CML (Kantarjian et al. 2002; Sawyers et al. 2002; Talpaz et al. 2002). As expected, outcomes were best among patients with chronic phase CML, compared with patients with more advanced disease (see Table 2). In addition, a randomized phase III trial (IRIS) comparing the safety and efficacy of imatinib vs IFN-alfa + cytarabine in 1106 patients with newly diagnosed CML demonstrated that not only was imatinib much better tolerated than IFN-alfa + cytarabine, it was also significantly more effective than IFN-alfa + cytarabine with regard to complete hematologic response rates (O'Brien et al. 2003), complete cytogenetic response rates (O'Brien et al. 2003), molecular responses (Hughes et al. 2003), and overall survival (Anstrom et al. 2004) (summarized in Table 3). Furthermore, baseline quality of life was maintained among patients treated with imatinib, whilst quality of life markedly deteriorated among patients treated with INF-alfa + cytarabine ( $P=0.001$, Hahn et al. 2003).

The efficacy of high-dose imatinib therapy in chronic phase CML has recently been investigated. The outcomes of these studies are summarized in Table 4. A small single-arm study conducted in patients with chronic phase CML who had failed treatment with IFN-alfa suggested that high-dose imatinib (400 mg twice a day) improved cytogenetic and molecular response rates without increasing toxicity, compared with responses typically achieved with standard-dose imatinib (400 mg once a day) (Cortes et al.
2003). These findings were subsequently confirmed in an independent study conducted in patients with newly diagnosed CML (Kantarjian et al. 2004). Dose escalation of imatinib to $600 \mathrm{mg}$ once a day or $400 \mathrm{mg}$ twice a day also appears to be effective in overcoming disease relapse/refractoriness in patients who have failed treatment with standard-dose imatinib (Kantarjian et al. 2003).

Imatinib has generally been well tolerated, even in high-dose trials. The majority of adverse events were of mild-to-moderate severity, and the most frequently reported adverse events were edema, nausea, diarrhea, muscle cramps, and rash. However, myelosuppression was more frequent in patients with accelerated phase or blast crisis CML and in patients treated with high-dose imatinib regimens.

In summary, extensive experience with imatinib in clinical trials has shown this agent to be highly effective and well tolerated in the treatment of CML. In common with other treatments for CML, best responses are achieved in patients with chronic phase disease; up to $90 \%$ of patients achieve complete cytogenetic remissions following treatment with high-dose imatinib, and up to $41 \%$ achieve molecular remissions in which BCR-ABL transcripts are undetectable by RT-PCR.

\section{Treatment guidelines}

Although guidelines for the treatment of $\mathrm{CML}$ are available (ASH 1999), these are out of date as they do not take into account the advances in treatment achieved with imatinib. Since the establishment of imatinib as first-line treatment for CML, one of the key dilemmas facing healthcare providers is how best to incorporate it into the treatment of patients who are candidates for potentially curative (but toxic) allo-SCT procedures (Deininger et al. 2003; Peggs \& Mackinnon 2003). Consequently, ASH is planning to update its existing guidelines in the near future (ASH online, accessed February 14, 2005).

\section{Cost of care}

Although several studies have investigated the cost-effectiveness of individual treatment strategies in specific patient subgroups (Kattan et al. 1996; Lee et al. 1998; Beck et al. 2001; Dalziel et al. 2004; Reed et al. 2004; Warren et al. 2004), few data are available regarding the overall economic and societal costs of $\mathrm{CML}$.

Table 4 | High-dose imatinib in patients with chronic myeloid leukemia in chronic phase

\begin{tabular}{|c|c|c|c|}
\hline \multirow{2}{*}{ Patient group } & \multicolumn{3}{|c|}{ Responses } \\
\hline & Hematologic & Cytogenetic & Molecular \\
\hline Failed treatment with interferon-alfa & $100 \%$ (complete) & $89 \%$ (complete) & $\begin{array}{c}56 \% \text { (BCR-ABL transcripts } \\
\text { undetectable in } 41 \%)\end{array}$ \\
\hline Newly diagnosed & Not reported & $90 \%$ (complete) & $\begin{array}{c}63 \% \text { (BCR-ABL transcripts } \\
\text { undetectable in } 28 \%)\end{array}$ \\
\hline \multirow[t]{2}{*}{ Hematologic resistance/relapse with standard dose imatinib } & $45 \%$ (complete) & $10 \%$ (partial) & Not reported \\
\hline & $20 \%$ (partial) & & \\
\hline Cytogenetic resistance/relapse with standard dose imatinib & & $56 \%$ (complete or partial) & Not reported \\
\hline
\end{tabular}




\section{Unmet needs}

Although most CML patients respond well to initial treatment with imatinib, resistance is a major problem. Indeed, a minority of previously untreated patients are resistant to imatinib from the outset (innate resistance), and the majority of those who initially respond to imatinib subsequently develop resistance (acquired resistance).

Treatment options for patients with imatinib-resistant CML are limited to allo-SCT, assuming the patient is eligible and a suitable donor is available, or investigational therapies, such as farnesyltransferase inhibitors (Hoover et al. 2002) or arsenic trioxide (La Rosee et al. 2002). Furthermore, the onset of imatinib resistance appears to correlate with disease progression and worsening prognosis (Shah et al. 2002). Therefore there is a need for novel agents to override imatinib resistance in CML.

An agent that produces complete cytogenetic and molecular responses, delays disease progression, and significantly improves survival expectation in imatinib-resistant $\mathrm{CML}$ patients would represent a major advance in the treatment of CML.

To develop new strategies to overcome imatinib resistance it is necessary to understand the underlying resistance mechanisms. Clinical resistance is primarily mediated by the acquisition of mutations within the BCR-ABL oncoprotein that prevent imatinib binding, and to a lesser extent by bcr-abl gene amplification. Other mechanisms of resistance that have been suggested include elevated levels of multidrug resistance protein, and overexpression of SRC kinases (Mahon et al. 2000; Nardi et al. 2004; Stone 2004).

The activation loop of ABL kinase oscillates between open (active) and closed (inactive) conformations. Imatinib can only bind when the activation loop is in the closed (inactive) conformation, locking the kinase in the inactive state (Schindler et al. 2000). At least 17 different amino acid substitutions within BCR-ABL have been identified that cause imatinib resistance by preventing imatinib binding (Hochhaus et al. 2002; Roche-Lestienne et al. 2002; Shah et al. 2002; von Bubnoff et al. 2002; Al-Ali et al. 2004). Some of these mutations directly affect the imatinib binding site. However, the majority of imatinib-resistance mutations occur in the activation loop, locking it into the open (active) conformation, which precludes imatinib binding.

Structural studies have demonstrated that pyriodol[2,3-D]pyrimidine dual SRC-ABL kinase inhibitors bind to BCR-ABL, inhibiting its kinase activity, regardless of whether the activation loop is in the open (active) or closed (inactive) conformation (Nagar et al. 2002). In principle, the dual SRC-ABL inhibitors, which have less stringent binding requirements than imatinib, could potentially override imatinib resistance due to their ability to bind to and inhibit ABL kinase when the activation loop is in the open (active) conformation.

The potential utility of dual SRC-ABL inhibitors to override imatinib resistance in $\mathrm{CML}$ is further supported by recent data indicating that imatinib resistance is associated with upregulated expression of the SRC kinase, Lyn (Donato et al. 2004; Wu et al. 2004).

\section{Drug review}

Dasatinib is a dual function SRC-ABL kinase inhibitor currently being developed for the treatment of patients with imatinib- resistant CML. The discovery of dasatinib is discussed in a recent review (Lombardo et al. 2004).

\section{Preclinical evidence}

\section{$X$-ray crystallography}

X-ray crystallography has confirmed that, like imatinib, dasatinib binds to the adenosine triphosphate binding site of ABL kinase with the central cores of the two drugs occupying overlapping regions, but extending in opposite directions. This study confirmed the ability of dasatinib to bind to ABL kinase when the activation loop is in the open (active) conformation, thus confirming the theoretical potential for dasatinib to override imatinib resistance. Furthermore, there were no apparent steric clashes to preclude dasatinib from binding when the activation loop is in the closed (inactive) conformation (Tokarski et al. 2004). This apparent ability of dasatinib to bind to $\mathrm{ABL}$ kinase in multiple conformational states indicates that it may have the potential for greater potency compared with imatinib.

Evidence for in vitro activity against imatinib-resistant $B C R-A B L$ isoforms

In vitro cell-based assays have demonstrated that dasatinib is more potent $(\times 2-\log )$ than imatinib in inhibiting unmutated BCR-ABL activity (Shah et al. 2004a). This increased potency may be due to the apparent ability of dasatinib to bind to $A B L$ kinase in multiple conformational states (Tokarski et al. 2004).

Dasatinib has also shown in vitro activity against 14 of 15 imatinibresistant cell lines expressing mutant $B C R-A B L$ isoforms, demonstrated by inhibition of kinase activity, and inhibition of cell growth (Shah et al. 2004a). The one imatinib-resistant cell line that was also resistant to dasatinib carried the T315I mutation, suggesting that this mutation confers cross resistance to both imatinib and dasatinib. The sensitivity of the different imatinibresistance mutations to treatment with dasatinib was varied. This suggests that, in the future, individual patient's BCR-ABL mutation status may guide decision making regarding the minimum serum concentrations of dasatinib required for therapeutic benefit.

In addition to the apparent ability of dasatinib to override imatinib resistance caused by mutations in BCR-ABL, recent in vitro data suggest that, like imatinib, dasatinib is selective for leukemic vs normal hematopoietic stem cells. Dasatinib $(5 \mathrm{nM})$ did not inhibit growth of bone marrow progenitors isolated from healthy volunteers, but did result in 60-80\% growth inhibition in bone marrow progenitors isolated from CML patients with unmutated $B C R-A B L$ or M351T imatinib-resistant BCR-ABL (Shah et al. 2004a).

A battery of pharmacogenomic biomarkers (other than BCR-ABL mutations) have also been identified, which predict sensitivity to dasatinib in tumor cell lines (Clark et al. 2005).

\section{Evidence of antileukemic activity in mouse models of imatinib-sensitive and imatinib-resistant $C M L$}

Models of BCR-ABL-dependent disease have been produced by injecting mice with cell lines that express unmutated BCR-ABL, or imatinib-resistant BCR-ABL isoforms (Shah et al. 2004a,b). Following administration of dasatinib (10 or $15 \mathrm{mg} / \mathrm{kg}$ ), 


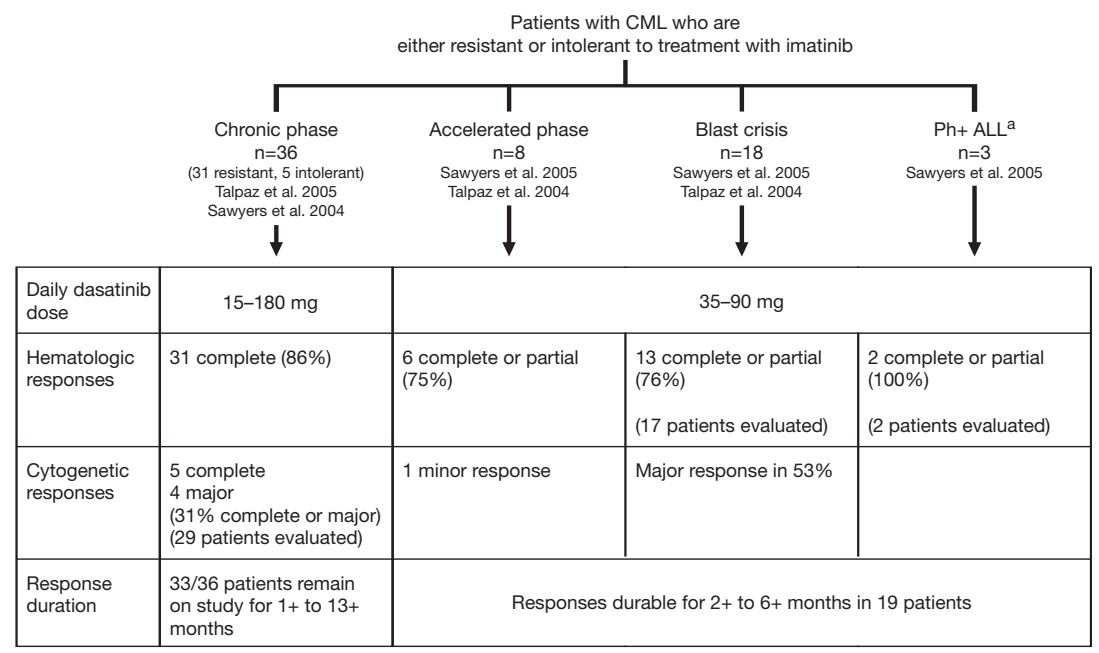

${ }^{\mathrm{a}} \mathrm{CML}$ in blast crisis where blasts have developed lymphoid morphology.
Fig. 5 | Phase I responses following treatment with dasatinib in patients with $\mathrm{Ph}+$, imatinib-resistant/intolerant chronic myeloid leukemia (CML) phosphorylation of CRKL (a BCR-ABL substrate) was inhibited for up to $7 \mathrm{~h}$, returning to baseline after $12 \mathrm{~h}$. Based on these preliminary data, a dose of $10 \mathrm{mg} / \mathrm{kg}$ was chosen for evaluation of dasatinib efficacy.

Three days after injecting the cell lines, mice were treated with vehicle or dasatinib for 2 weeks. All vehicle-treated mice developed progressive disease, with massive hepatic and splenic infiltration, which resulted in death in around 15 days. Conversely, dasatinibtreated mice with unmutated BCR-ABL tumors or M351T (imatinibresistant) tumors appeared to be healthy (no weight loss, lethargy, or ruffled fur), and showed a $>1$-log decrease in BCR-ABL kinase activity (measured by bioluminescence assay), normal spleen weight, and significantly longer survival compared with vehicletreated animals. Consistent with in vitro studies, mice with T315I BCR-ABL tumors did not respond to treatment with dasatinib (no significant reduction in kinase activity by bioluminescence assay) and showed no significant improvement in survival compared with vehicle-treated animals.

Treatment with dasatinib at doses as low as $5 \mathrm{mg} / \mathrm{kg}$ per day has been shown to be curative in other murine xenograft models of imatinib-resistant CML (Lee et al. 2004).

\section{Evidence for antileukemic activity in a mouse model of intracranial CML}

Growing evidence suggests that imatinib is a poor penetrator of the blood-brain barrier, resulting in subtherapeutic levels of the drug in the central nervous system. Several clinical cases have been reported in which central nervous system relapse has occurred despite complete hematologic responses in the blood and bone marrow (Leis et al. 2004). Furthermore, in at least one animal model of CML, imatinib has shown limited ability to cross the blood-brain barrier, allowing the central nervous system to act as a sanctuary for leukemic cells (Wild et al. 2004).

It has been suggested that the limited ability of imatinib to cross the blood-brain barrier may be due to the fact that it is a substrate for
P-glycoprotein. In contrast, dasatinib is not a P-glycoprotein substrate, and is therefore more likely to achieve therapeutic levels in the central nervous system. To test this hypothesis a mouse model of intracranial CML was created by transplanting K562 CML tumors intracranially. Mice were treated with dasatinib twice daily for up to 40 days. Tumor regression was achieved at a dasatinib dose of $15 \mathrm{mg} / \mathrm{kg}$, with complete stasis of intracranial tumor growth during therapy. In addition, survival was increased by 450 and $268 \%$ at dose levels of 15 and $5 \mathrm{mg} / \mathrm{kg}$, respectively. These preliminary data indicate that dasatinib may potentially offer advantages over imatinib in the management of intracranial CML (Wild et al. 2004).

\section{Pharmacokinetic profile in mice}

In a model of BCR-ABL-dependent disease, oral dasatinib was curative over a range of doses $(1.25-50 \mathrm{mg} / \mathrm{kg})$. At the minimum effective dose of $1.25 \mathrm{mg} / \mathrm{kg}$, maximum inhibition of BCR-ABL activity was observed $3 \mathrm{~h}$ after dosing, with complete recovery of BCR-ABL activity in 7-17 $\mathrm{h}$. This model predicted that a minimum plasma concentration of $20 \mathrm{nM}$ is required to effectively inhibit BCR-ABL activity. In addition, while a $1.25 \mathrm{mg} / \mathrm{kg}$ per day dose was curative with twice-daily dosing, a higher $5 \mathrm{mg} / \mathrm{kg}$ per day dose was required for equivalent efficacy with once-daily dosing, suggesting that twice-daily dosing regimens may be more effective than once daily (Luo et al. 2004).

\section{Clinical evidence}

Patients with CML who are resistant or intolerant to previous treatment with imatinib were enrolled onto a phase I dose escalation study (Fig. 5). This study included 36 patients with chronic phase $\mathrm{CML}$, eight patients with accelerated phase CML, 18 patients in blast crisis, and three patients with $\mathrm{Ph}+\mathrm{ALL}$. Patients with chronic phase CML were treated with dasatinib at doses of $15-180 \mathrm{mg} /$ day (as a single dose or split into two equal doses) for 5-7 days/week, whilst patients in the accelerated phase or blast crisis were treated with dasatinib at doses of 35-90 mg/day twice daily. Responses according to phase of disease are summarized below. 


\section{Phase I responses: patients with chronic phase CML}

To date 36 patients with chronic phase CML enrolled onto this phase I study (imatinib resistant $n=31$, imatinib intolerant $n=5, B C R$ $A B L$ mutations associated with imatinib resistance $n=27$ ) have been followed for $>4$ weeks and were evaluable for response (Sawyers et al. 2004; Talpaz et al. 2005).

Thirty-one patients (86\%) achieved a complete hematologic response. Only two patients experienced disease progression: one of whom carried the imatinib resistance mutation, T315I, which was also associated with resistance to dasatinib in preclinical studies.

Twenty-nine patients were evaluated cytogenetically: 13 demonstrated cytogenetic improvement, including five complete and four major cytogenetic responses. Hematologic and cytogenetic responses were achieved in patients with or without BCR-ABL mutations (Sawyers et al. 2004; Talpaz et al. 2005; Shah et al. 2005). Furthermore, these responses were durable with 33 of 36 patients remaining on study for $1+$ to $13+$ months (Talpaz et al. 2005).

BCR-ABL transcripts were monitored by quantitative RT-PCR in a subset of 13 patients treated with dasatinib (Shah et al. 2004c). Four patients (three imatinib resistant, two of whom carried the known imatinib-resistance mutation, M351T) achieved a major cytogenetic response, which corresponded with 1- or 2-log reductions in $B C R-A B L$ transcript levels, and an overall $32 \%$ median reduction in $B C R-A B L$ transcripts after 4 weeks of treatment. In contrast, none of the nine patients who failed to achieve a major cytogenetic response achieved a 1-log reduction in BCR-ABL transcript levels.

These preliminary data therefore indicate that the achievement of a major cytogenetic response is associated with 1- or 2-log reduction in BCR-ABL transcript expression. In addition, quantitative RT-PCR appears to offer a rapid and reliable method of monitoring disease burden and response to therapy in this setting (Shah et al. 2004c). It is worthy of note that similar reductions in BCR-ABL transcript levels have previously correlated with cytogenetic responses achieved with imatinib (Shah et al. 2004c).

Responses in patients with accelerated phase CML,

blast crisis, or $P h+A L L$

To date, 29 patients with accelerated phase CML $(n=8)$, blast crisis $(n=18)$, or $\mathrm{Ph}+\operatorname{ALL}(\mathrm{n}=3)$ who entered this phase I trial have received treatment with dasatinib (Talpaz et al. 2004; Sawyers et al. 2005). Of 28 patients for whom BCR-ABL mutation status was known, 16 had mutations known to confer imatinib resistance. Hematologic response rates (complete and partial) were as follows: $75 \%$ (six of eight) in patients with accelerated phase CML, $76 \%$ (13 of 17 ) in patients in blast crisis, and 100\% (two of two) in patients whose disease had transformed to Ph+ ALL. These responses were durable for $2+$ to $6+$ months in 19 patients (Sawyers et al. 2005).

Among patients in blast crisis, 53\% achieved a major cytogenetic response. Of note, the $\mathrm{T} 315 \mathrm{I}$ mutation, which conferred cross resistance to both imatinib and dasatinib in preclinical models, was identified in four of six patients who were resistant to dasatinib therapy.

\section{Safety and tolerability}

Dasatinib treatment was generally well tolerated regardless of disease phase. In patients with chronic phase CML, three of 36 developed grade 4 thrombocytopenia and two of 36 developed grade 4 neutropenia, all of which were reversible and easily managed with dose modification. One patient developed a duodenal ulcer, and mild QTc prolongation was noted (Talpaz et al. 2005). In patients with advanced disease, eight of 29 developed grade 4 thrombocytopenia, one patient developed grade 3/4 fluid retention, and two patients developed tumor lysis syndrome (Sawyers et al. 2005).

SRC inhibitors are known to be important modulators of T-cell activation. It is therefore noteworthy that a recent report indicated that dasatinib induces complete hematologic remission without affecting the ability of $\mathrm{CD}^{+}$and $\mathrm{CD}^{+} \mathrm{T}$ cells to produce Th1 and Th2 cytokines when stimulated (Gao et al. 2005).

Overall, these preliminary phase I data indicate that dasatinib is frequently able to override imatinib resistance in patients with chronic phase disease and those with more advanced disease (accelerated phase or blast crisis). The drug also appears to be well tolerated, regardless of the disease phase. The maximum tolerated dose has yet to be established and dose escalation continues to establish the dose range that yields optimal results.

Based on these encouraging data, phase II studies of dasatinib in the treatment of patients with imatinib-resistant CML are planned.

\section{Resource utilization}

Dasatinib is still in the preliminary stages of clinical development, and its future use in the treatment of CML will depend upon the outcomes of phase II and III trials. However, the available preliminary data suggest that it may potentially provide a much needed therapeutic option for patients with imatinib-resistant CML. The future use of dasatinib in the treatment of CML is likely to depend on its safety profile. In particular, SRC kinase inhibition is associated with immunosuppression, which raises the possibility that treatment with dasatinib might be associated with more adverse events than imatinib (Doggrell 2005). Long-term follow-up data will therefore be required to fully assess the safety profile of dasatinib.

In a screen for $\mathrm{BCR}-\mathrm{ABL}$ mutations conferring resistance to imatinib and dasatinib, the mutation profiles conferring resistance to the two drugs were different and 30-50-fold fewer mutant clones were resistant to both imatinib and dasatinib, compared with each drug individually (Burgess et al. 2004). This suggests that dasatinib in combination with imatinib could potentially be useful in the firstline management of CML, particularly with regard to delaying the development of drug resistance.

In addition, given the apparent potency of dasatinib, its use in combination with other agents known to be active in CML could potentially be an effective strategy for purging residual disease and achieving molecular remissions.

\section{Drug profile}

Although imatinib has proven to be extremely effective in first-line treatment of CML, producing hematologic, cytogenetic, and molecular remissions, resistance to the drug frequently develops. 
Imatinib resistance is problematic for two reasons: alternative therapeutic options are extremely limited, and the onset of imatinib resistance is usually associated with disease progression and worsening prognosis. There is therefore a need for new treatment strategies to be developed to override imatinib resistance in CML.

Dasatinib is an orally active SRC-ABL inhibitor that is currently in development for the treatment of imatinib-resistant CML. This compound has shown potent antileukemic activity in cell lines expressing imatinib-resistant BCR-ABL isoforms, and striking remissions and significant prolongation of survival in a series of xenograft mouse models of imatinib-sensitive and imatinibresistant CML. Furthermore, dasatinib has shown promising in vitro activity against malignant bone marrow cells isolated from patients with imatinib-resistant CML. The apparent ability of dasatinib to cross the blood-brain barrier in mice suggests that it might also prove useful in the treatment of patients with intracranial CML.

Preliminary evidence from the initial phase I dose escalation study is encouraging, indicating that dasatinib can induce hematologic and cytogenetic responses in patients with imatinib-resistant CML in all phases of the disease. However, although dasatinib appears to be able to override the majority of BCR-ABL mutations that cause imatinib resistance, it does not inhibit at least one widespread imatinib-resistant BCR-ABL isoform (T315I) (Shah et al. 2005). Dasatinib appears to be generally well tolerated, regardless of disease phase, and long-term tolerability data are eagerly awaited.

Although dasatinib is still in the initial stages of clinical development, early evidence suggests that its potential impact on the treatment of CML could be substantial. Not only could dasatinib provide a much needed treatment option for patients with imatinibresistant CML, but also, combined with imatinib, it might prove to be useful in delaying the onset of resistance seen with imatinib monotherapy. Furthermore, dasatinib, in combination with other agents known to be active in CML, may also prove useful for purging the residual leukemic cells that persist even in patients whose disease is controlled by imatinib.

\section{References}

Ahuja H, Bar-Eli M, Arlin Z, et al. Alterations in the p53 gene and the clonal evolution of the blast crisis of chronic myelocytic leukemia. PNAS USA. 1989;86:6783-6787.

Al Ali HK, Heinrich MC, Lange T, et al. High incidence of BCR-ABL kinase domain mutations and absence of mutations of the PDGFR and KIT activation loops in CML patients with secondary resistance to imatinib. Hematol J. 2004;5(1):55-60.

Anstrom KJ, Reed SD, Allen AS, Glendenning GA, Schulman KA. Long-term survival estimates for imatinib versus interferon-alpha plus low-dose cytarabine for patients with newly diagnosed chronic phase chronic myeloid leukemias. Cancer. 2004;101(11):2584-2592.

ASH (American Society of Hematology). An evidence-based analysis of the effect of busulfan, hydroxyurea, interferon, and allogeneneic bone marrow transplantation in treating the chronic phase of chronic myeloid leukaemia. Blood. 1999;95(5):1517-1536.

Beck JR, Guilhot J, Giles FJ, Aoki N, Wirt DP, Guilhot F. Cytarabine added to interferon improves the cost-effectiveness of initial therapy for patients with early phase chronic myelogenous leukaemia. Leuk Lymphoma. 2001;41(1-2):117-124.

Besa EC. Chronic myelogenous leukaemia. eMedicine, September 92004. http://www.emedicine.com/med/topic371.htm
Bleakley M, Riddell SR. Molecules and mechanisms of the graft-versus-leukemia effect. Nat Rev Cancer. 2004;4(5):371-380.

Burgess MR, Shah NP, Skaggs BJ, Lee FY, Sawyers CL. Comparative analysis of two BCR-ABL small molecule inhibitors reveals overlapping but distinct mechanisms of resistance. ASH 2004 oral presentation (Abstract 552).

Clark E, Reeves KA, Han X, et al. Identification of pharmacogenomic markers for predicting sensitivity to BMS-354825, a SRC/ABL kinase inhibitor. 2005 American Society of Clinical Oncology (ASCO) Annual Meeting, Abstract 3010.

Clift RA, Storb R. Marrow transplantation for CML: the Seattle experience. BMT. 1996;17(Suppl. 3):S1-S3.

Clift RA, Radich J, Appelbaum FR, et al. Long-term follow-up of a randomized study comparing cyclophosphamide and total body irradiation with busulfan and cyclophosphamide for patients receiving allogeneic marrow transplants during chronic phase myeloid leukemia. Blood. 1999;94:3960-3962.

Cortes JE, Talpaz M, Kantarjian H. Chronic myelogenous leukemia: a review. Am J Med. 1996;100(5):555-570.

Cortes J, Kantarjian HM, Giralt S, Talpaz M. Natural history and staging of chronic myelogenous leukemia. Baillieres Clin Haematol. 1997;10(2):277-290.

Cortes J, Giles F, O'Brien S, et al. Result of high dose imatinib mesylate in patients with Philadelphia chromosome-positive chronic myeloid leukemia after failure of interferon-alfa. Blood. 2003;102:83-86.

Dalziel K, Round A, Stein K, Garside R, Price A. Effectiveness and costeffectiveness of imatinib for first-line treatment of chronic myeloid leukaemia in chronic phase: a systematic review and economic analysis. Health Technol Assess. 2004;28(3):1-120.

Deininger MW, O'Brien SG, Ford JM, Druker BJ. Practical management of patients with chronic myeloid leukemia receiving imatinib. $J$ Clin Oncol. 2003;21:1637-1647.

Derderian PM, Kantarjian HM, Talpaz M, et al. Chronic myelogenous leukemia in the lymphoid blastic phase: characteristics, treatment response and prognosis. Am J Med. 1993;94:69-74.

Doggrell SA. BMS-354825: a novel drug with potential for the treatment of imatinib-resistant chronic myeloid leukaemia. Expert Opin Invest Drugs. 2005;14(1):89-91.

Donato NJ, Wu J, Kong LY, Lee F, Talpaz M. The SRC/ABL inhibitor BMS-354825 overcomes resistance to imatinib mesylate in chronic myelogenous leukaemia cells through multiple mechanisms. ASH 2004 poster (Abstract 1989).

Druker BJ, Tamura S, Buchdunger E, et al. Effects of a selective inhibitor of the Abl tyrosine kinase on growth of Bcr-Abl positive cells. Nat Med. 1996;2:561-566.

Druker BJ, Talpaz M, Resta DJ, et al. Efficacy and safety of specific inhibitor of Bcr-Abl tyrosine kinase in chronic myeloid leukemia. $N$ Engl $J$ Med. 2001a;344:1031-1037.

Druker BJ, Sawyers CL, Kantarjian H, et al. Activity of a specific inhibitor of the Bcr-Abl tyrosine kinase in the blast crisis of chronic myeloid leukemia and acute lymphoblastic leukemia with the Philadelphia chromosome. N Engl J Med. 2001b;344:1038-1042.

Faderl S, Talpaz M, Estror Z, O'Brien S, Kurzrock R, Kantarjian HM. The biology of chronic myeloid leukemia. N Engl J Med. 1999;341(3):164-172.

Gao H, Talpaz M, Lee BN, et al. BMS-354825 induced complete hematologic remission in chronic phase $C M L$ patients without affecting T-cell cytokine production. 2005 American Society of Clinical Oncology (ASCO) Annual Meeting, Abstract 6619.

Goldman J. ABC of clinical haematology. Chronic myeloid leukemia. BMJ. 1997;314:657-660.

Gratwohl A, Hermans J. Allogeneic bone marrow transplantation for chronic myeloid leukemia. Working Party Chronic Leukemia of the European Group for Blood and Marrow Transplantation (EBMT). BMT. 1996;17(Suppl. 3):S7-S9.

Gratwohl A, Hermans J, Goldman JA, et al. Risk assessment for patients with chronic myeloid leukaemia before allogeneic blood or marrow transplantation. Chronic Leukemia Working Party of the European Group for Blood and Marrow Transplantation. Lancet. 1998;352:1087-1092. 
Griffin JD, Todd RF 3rd, Ritz J, et al. Differentiation patterns in the blastic phase of chronic myeloid leukemia. Blood. 1983;61:85-91.

Guilhot $\mathrm{F}$, Chastang $\mathrm{C}$, Michallet $\mathrm{M}$, et al. Interferon alfa-2b combined with cytarabine versus interferon alone in chronic myelogenous leukemia. French Myeloid Leukemia Study Group. N Engl J Med. 1997;337:223-229.

Hahn EA, Glendenning GA, Sorensen MV, et al. Quality of life in patients with newly diagnosed chronic phase chronic myeloid leukemia on imatinib versus interferon alfa plus low dose cytarabine: results from the IRIS study. $J$ Clin Oncol. 2003;21:2138-2146.

Hansen JA, Gooley TA, Martin PJ, et al. Bone marrow transplants from unrelated donors for patients with chronic myeloid leukemia. N Engl $J$ Med. 1998;338:962-968.

Hehlmann R, Heimpel H, Hasford J, et al. Randomized comparison of busulfan and hydroxyurea in chronic myelogenous leukemia: prolongation of survival by hydroxyurea. The German CML Study Group. Blood. 1993;82:398-407.

Hehlmann R, Heimpel H, Hasford J, et al. Randomized comparison of interferonalpha with busulfan and hydroxyurea in chronic myelogenous leukemia. The German CML Study Group. Blood. 1994;84:4064-4077.

Hill JM, Meehan KR. Chronic myelogenous leukemia. Postgrad Med. 1999;106(3):online. (www.postgradmed.com/issues/1999/09_99/hill.htm) Hochhaus A, Kreil S, Corbin AS, et al. Molecular and chromosomal mechanisms of resistance to imatinib (STI571) therapy. Leukemia. 2002;16(11):2190-2196.

Hoover RR, Mahon FX, Melo JV, Daley GO. Overcoming STI571 resistance with the farnesyltransferase inhibitor SCH66336. Blood. 2002;100:1068-1071.

Horowitz MM, Rowlings PA, Passweg JR, et al. Allogeneic bone marrow transplantation for CML: a report from the International Bone Marrow Transplant Registry. BMT. 1996;17(Suppl. 3):S5-S6.

Hughes TP, Kaeda J, Branford S, et al. Frequency of major molecular responses to imatinib or interferon-alfa plus cytarabine in newly diagnosed chronic myeloid leukemia. N Engl J Med. 2003;349:1423-1432.

ICSGCML (Italian Cooperative Study Group on Chronic Myeloid Leukemia). Interferon-alfa as compared with conventional chemotherapy for the treatment of chronic myeloid leukemia. N Engl J Med. 1994;330:820-825.

ICSGCML (Italian Cooperative Study Group on Chronic Myeloid Leukemia). Longterm follow-up of the Italian trial of interferon-alpha versus conventional chemotherapy in chronic myeloid leukemia. Blood. 1998;92:1541-1548.

Jemal A, Thomas A, Murray T, Thun M. Cancer Statistics 2002. CA Cancer J Clin. 2002;52:23-47.

John AM, Thomas NS, Mufti GJ, Padua RA. Targeted therapies in myeloid leukemia. Semin Cancer Biol. 2004;14:41-62.

Kantarjian HM, Keating MJ, Talpaz M, et al. Chronic myelogenous leukemia in blast crisis: analysis of 242 patients. Am J Med. 1987;83:445-454.

Kantarjian HM, Smith TL, O'Brien S, Beran M, Pierce S, Talpaz M. Prolonged survival in chronic myelogenous leukemia after cytogenetic response to interferon-alfa therapy. The Leukemia Service. Ann Intern Med.

1995;122:254-261.

Kantarjian HM, Giles FJ, O'Brien SM, Talpaz M. Clinical course and therapy of chronic myelogenous leukemia with interferon-alpha and chemotherapy. Hematol Oncol Clin North Am. 1998;12:31-80.

Kantarjian HM, Talpaz M, Smith TL, et al. Homoharringtonine and low dose cytarabine in the management of late chronic phase chronic myelogenous leukemia. J Clin Oncol. 2000;18:3513-3521.

Kantarjian HM, Talpaz M, Santini V, Murgo A, Cheson B, O'Brien S. Homoharringtonine: history, current research and future direction. Cancer. 2001;92:1591-1605.

Kantarjian $\mathrm{H}$, Sawyers $\mathrm{C}$, Hochhaus A, et al. Hematologic and cytogenic responses to imatinib mesylate in chronic myelogenous leukemia. $N$ Engl J Med. 2002;346(9):645-652.

Kantarjian HM, Talpaz M, O'Brien S, et al. Dose escalation of imatinib mesylate can overcome resistance to standard dose therapy in patients with chronic myelogenous leukemia. Blood. 2003;101:473-475.
Kantarjian H, Talpaz M, O'Brien S, et al. High-dose imatinib mesylate therapy in newly diagnosed Philadelphia chromosome-positive chronic phase chronic myeloid leukemia. Blood. 2004;103(8):2873-2878.

Kattan MW, Inoue Y, Giles FJ, et al. Cost-effectiveness of interferon-alpha and conventional chemotherapy in chronic myelogenous leukemia. Ann Intern Med. 1996;125(7):541-548.

La Rosee P, Johnson K, O'Dwyer ME, Druker BJ. In-vitro studies of the combination of imatinib mesylate (Gleevec) and arsenic trioxide (Trisenox) in chronic myelogenous leukemia. Exp Hematol. 2002;30:729-737.

Lee SJ, Anasetti C, Kuntz KM, Patten J, Antin JH, Weeks JC. The costs and cost-effectiveness of unrelated donor bone marrow transplantation for chronic phase chronic myelogenous leukemia. Blood. 1998;92(11):4047-4052.

Lee FY, Lombardo L, Borzilleri R, et al. BMS-354825-A potent dual SRC/ABL kinase inhibitor possessing curative efficacy against imatinib sensitive and resistant human CML models in vivo. AACR. 2004 (Abstract 3987).

Leis JF, Stepan DE, Curtin PT, et al. Central nervous system failure in patients with chronic myelogenous leukaemia, lymphoid blast crisis and Philadelphia chromosome positive acute lymphoblastic leukaemia treated with imatinib (STI571). Leuk Lymphoma. 2004;45(4):695-698.

Lombardo LJ, Lee FY, Chen P, et al. Discovery of N-(2-chloro-6-methyl-phenyl)-2(6-(4-(2-hydroxyethyl)-piperazin-1-yl)-2-methylpyrimidin-4-ylamino)thiazole-5carboxamide (BMS-354825), a dual Src/Abl kinase inhibitor with potent antitumor activity in preclinical assays. J Med Chem. 2004;47(27):6658-6661

Luo FR, Yang Z, Camuso A, et al. Pharmacokinetics- and pharmacodynamicsguided optimization of the dose and treatment schedule for the dual SRC/ABL inhibitor BMS-354825. ASH 2004 poster (Abstract 1987).

Mahon FX, Deininger MW, Schultheis B, et al. Selection and characterization of $B C R-A B L$ positive cell lines with differential sensitivity to the tyrosine kinase inhibitor STI571: diverse mechanisms of resistance. Blood. 2000:96:1070-1079.

Maness LJ, McSweeney PA. Treatment options for newly diagnosed patients with chronic myeloid leukemia. Curr Hematol Reports. 2004;3:54-61.

Mitelman F. The cytogenetic scenario of chronic myeloid leukemia. Leuk Lymphoma. 1993;11(Suppl. 1):11-15.

Nagar B, Bornmann WG, Pellicena P, et al. Crystal structures of the kinase domain of c-Abl in complex with the small molecule inhibitors PD173955 and imatinib (STI-571). Cancer Res. 2002;62(15):4236-4243.

Nardi V, Azam M, Daley GO. Mechanisms and implications of imatinib resistance mutations in BCR-ABL. Curr Opin Hematol. 2004;11(1):35-43.

Nowell PC, Hungerford DA. A minute chromosome in human chronic granulocytic leukemia. Science. 1960;132:1497.

O'Brien S, Kantarjian H, Keating M, et al. Homoharringtonine therapy induces responses in patients with chronic myelogenous leukemia in late chronic phase. Blood. 1995;86(9):3322-3326.

O'Brien SG, Guilhot F, Larson RA, et al. Imatinib compared with interferon and low dose cytarabine for newly diagnosed chronic phase chronic myeloid leukemia. N Engl J Med. 2003;348:994-1004.

Or R, Shapira MY, Resnick I, et al. Nonmyeloablative allogeneic stem cell transplantation for the treatment of chronic myeloid leukemia in first chronic phase. Blood. 2003;101:441-445.

Peggs K, Mackinnon S. Imatinib mesylate-the new gold standard for treatment of chronic myeloid leukemia. N Engl J Med. 2003;348:1048-1050.

Reed SD, Anstrom KJ, Ludmer JA, Glendenning GA, Schulman KA. Costeffectiveness of imatinib versus interferon-alpha plus low-dose cytarabine for patients with newly diagnosed chronic-phase chronic myeloid leukemia. Cancer. 2004;101(11):2574-2583.

Roche-Lestienne C,Soenan-Cornu V, Grardel-Duflos N, et al. Several types of mutations of the Abl gene can be found in chronic myeloid leukaemia patients resistant to STI571, and they can pre-exist to the onset of treatment. Blood. 2002;100(3):1014-1018.

Rowley JD. A new consistent chromosomal abnormality in chronic myelogenous leukemia identified by quinacrine fluorescence and Giemsa staining. Nature. 1973;243:290-293. 
Sawyers CL, Hochhaus A, Feldman E, et al. Imatinib induces hematologic and cytogenetic responses in patients with chronic myelogenous leukaemia in myeloid blast crisis: results of a phase II study. Blood. 2002; 99(10):3530-3539.

Sawyers CL, Shah NP, Kantarjian HM, et al. Hematologic and cytogenetic responses in imatinib-resistant chronic phase chronic myeloid leukaemia patients treated with the dual SRC/ABL kinase inhibitor BMS-354825: Results form a phase I dose escalation study. ASH 2004 plenary oral presentation (Abstract 1).

Sawyers CL, Shah NP, Kantarjian HM, et al. A phase / study of BMS-354825 in patients with imatinib-resistant and intolerant accelerated and blast phase chronic myeloid leukaemia (CML): results from CA180002. 2005 American Society of Clinical Oncology (ASCO) Annual Meeting, Abstract 6520.

Schindler T, Bornmann W, Pellicena P, Miller WT, Clarkson B, Kuriyan J. Structural mechanism for STI-571 inhibition of abelson tyrosine kinase. Science. 2000;289(5486):1938-1942.

Shah NP, Nicoll JM, Nagar B, et al. Multiple BCR-ABL kinase domain mutations confer polyclonal resistance to the tyrosine kinase inhibitor imatinib (STI571) in chronic phase and blast crisis chronic myeloid leukaemia. Cancer Cell. 2002;2:117-125.

Shah NP, Tran C, Lee FY, Chen P, Norris D, Sawyers CL. Overriding imatinib resistance with a novel ABL kinase inhibitor. Science. 2004a;305:399-401.

Shah NP, Tran C, Lee FY, Sawyers CL. BMS-354825 is a novel orally bioavailable small molecule $A B L$ tyrosine kinase inhibitor that successfully and safely inhibits the kinase activity of multiple imatinib-resistant BCR-ABL isoforms in vitro and in vivo. AACR. 2004b (Abstract 5624).

Shah NP, Branford S, Hughes TP, Nicoll JM, Decillis AP, Sawyers CL. Major cytogenetic responses to BMS-354825 in patients with chronic myeloid leukaemia are associated with one or two log reduction in BCR-ABL transcript. ASH $2004 \mathrm{C}$ poster (Abstract 1008).

Shah N, Sawyers CL, Kantarjian HM, et al. Correlation of clinical response to $B M S-354825$ with BCR-ABL mutation status in imatinib-resistant patients with chronic myeloid leukaemia (CML) and Philadelphia chromosome-associated acute lymphoblastic leukaemia (Ph+ ALL). 2005 American Society of Clinical Oncology (ASCO) Annual Meeting, Abstract 6521.

Silver TR, Woolf SH, Hehlmann R, et al. An evidence-based analysis of the effect of busulphan, hydroxyurea, interferon and allogeneic bone marrow transplantation in treating the chronic phase of chronic myeloid leukemia: developed for the American Society of Hematology. Blood. 1999;94:1517-1536.

Stone RM. Optimizing treatment of chronic myeloid leukemia: a rational approach. Oncologist. 2004;9:259-270.
Talpaz M, Silver RT, Druker BJ, et al. Imatinib induces durable hematologic and cytogenetic responses in patients with accelerated phase chronic myeloid leukemia: results of a phase 2 study. Blood. 2002; 99(6):1928-1937.

Talpaz M, Kantarjian H, Shah NP, et al. Hematologic and cytogenetic responses in imatinib-resistant accelerated and blast phase chronic myeloid leukaemia (CML) patients treated with the dual SRC/ABL kinase inhibitor BMS-354825: Results from a phase I dose escalation study. ASH 2004 oral presentation (Abstract 20).

Talpaz M, Kantarjian HM, Paquette R, et al. A phase I study of BMS-354825 in patients with imatinib-resistant and intolerant chronic phase chronic myeloid leukaemia (CML): results from CA180002. 2005 American Society of Clinical Oncology (ASCO) Annual Meeting, Abstract 6519.

Tokarski JS, Newitt J, Lee FY, et al. The crystal structure of Abl kinase with BMS354825, a dual SRC/ABL kinase inhibitor. ASH 2004 (oral presentation) (Abstract 553).

Travis J. Gleevec, chapter two: new leukemia drug aims to overcome resistance. Science. 2004;305:319-320.

Vardiman JW, et al. Chronic myeloproliferative diseases and myelodysplastic myeloproliferative diseases. In: Jaffe ES, Harris NL, Stein H, Vardiman JW, editors. WHO classification of Rumours. Pathology and Genetics. Tumours of Haematopoietic and Lymphoid Tissues. Lyon, France: IARC Press; 2001, pp. 17-31 and 47-52.

von Bubnoff N, Schneller F, Peschel C, Duyster J. BCR-ABL gene mutations in relation to clinical resistance of Philadelphia-chromosome-positive leukaemia to STI571: a prospective study. Lancet. 2002;359(9305):487-491.

Warren E, Ward S, Gordois A, Scuffham P. Cost utility analysis of imatinib mesylate for the treatment of chronic myelogenous leukaemia in the chronic phase. Clin Ther. 2004;26(11):1924-1933.

Weisdorf DJ, Anasetti C, Antin JH, et al. Allogeneic bone marrow transplantation for chronic myelogenous leukemia: comparative analysis of unrelated versus matched sibling donor transplantation. Blood. 2002;99:1971-1977.

Wild R, Castaneda S, Flefleh C, et al. BMS-354825, a dual SRC/ABL kinase inhibitor, displays potent anti-tumor activity in a model of intracranial CML growth. ASH 2004 poster (Abstract 1988).

Wu JY, Donato NJ, Hong DS, Lee FY, Talpaz M. The SRC/ABL kinase inhibitor BMS354825 induces apoptosis and overcomes imatinib resistance in chronic myelogenous leukemia cell lines and patient specimens. AACR 2004 (Abstract 3850).

Correspondence: Sonya Haslam, Core Medical Publishing, Mere House, Brook Street, Knutsford, Cheshire WA16 8GP, UK or at editor@coreevidence.com 